\title{
LA COLABORACIÓN ENTRE JULIO ANTONIO (1889-1919) Y SEBASTIÁN MIRANDA (1885-1975) Y SUS PROYECTOS PARA MONUMENTOS CONMEMORATIVOS
}

\author{
POR \\ María Soto CANO \\ Dra. en Historia del Arte
}

\begin{abstract}
En el presente artículo se analiza la relación de amistad y colaboración de los escultores españoles Julio Antonio y Sebastián Miranda, así como los tres proyectos monumentales que de ellas nacieron, todo dentro del contexto artístico del primer tercio del siglo XX español.

Palabras clave: Escultura conmemorativa; Proyectos; Siglo XX; Julio Antonio; Sebastián Miranda.

In this article the author analyzes the relationship of friendship and collaboration between the two Spanish sculptors Julio Antonio and Sebastián Miranda, as well as the three monumental projects born from this association, within the artistic context of the first third of $20^{\text {th }}$ century Spain.
\end{abstract}

Key words: Commemorative Sculpture; Projects; $20^{\text {th }}$ Century; Julio Antonio; Sebastián Miranda.

“(...) Esto, es decir, un retorno al arte clásico, cuya expresión por antonomasia fue la escultura, y otras cosas que sería prolijo enunciar, representa el proyecto de Julio Antonio y Sebastián Miranda”.

Ramón PÉRez DE AyAlA,

"Julio Antonio y Sebastián Miranda", Gran Mundo, Madrid, 15-3-1914.

Julio Antonio (Antonio Rodríguez Hernández, 1889-1919) y Sebastián Miranda (1885-1975) fueron dos destacados escultores en el panorama del siglo XX español, aunque más conocido el primero de ellos. Durante la segunda década de la pasada centuria y hasta la muerte de Julio Antonio en 1919, mantuvieron una estrecha relación de amistad y una colaboración que se materializó en tres proyectos monumentales, objeto de análisis en el presente artículo.

Julio Antonio (fig. 1) ha sido considerado como uno de los renovadores de la escultura española contemporánea. Nacido en Mora de Ebro (Tarragona) en 1889, murió prematuramente 
en Madrid en 1919. Se consagró como artista ya desde mediados de la década de 1910 y su figura y su obra han sido objeto de numerosos estudios ${ }^{1}$. Artista viajero y bohemio, enamorado de Italia, de la antigüedad clásica y del renacimiento italiano, especialmente de Donatello y Miguel Ángel, inició una revisión de la escultura española a través de la recuperación del arte clásico y de una nueva atención hacia los personajes populares por medio de una representación sobria y realista. Sus obras más conocidas son el grupo de los Bustos de la raza y el Mausoleo Lemonier que, al exponerse poco después de su muerte, consiguió generar el aprecio popular por su producción. Sin embargo, no fue menos importante su aportación a la escultura monumental. En ella intentó impulsar una nueva concepción a través de la integración de este arte con la arquitectura, de las referencias clásicas y de un simbolismo e idealismo de raíz también clásica.

Sebastián Miranda (fig. 2) es, al contrario que Julio Antonio, un escultor aún poco conocido². Nacido en Oviedo en 1885 y muerto en Madrid en 1975, se dedicó durante casi toda su vida al género de la retratística de pequeño formato. Esto ha hecho que su obra permanezca dispersa y desconocida en numerosas colecciones particulares. Compartía con Julio Antonio su devoción por la antigüedad clásica, fruto de sus estudios en el Instituto de Bellas Artes de Roma, y su pasión por representar a los tipos más variados de la geografía española. Ejemplo de esto último es su obra más conocida, El Retablo del Mar. Su producción en la segunda década del siglo XX, de tintes caricaturescos y expresionistas, se vincula con la corriente humorista defendida por José Francés y con las figuras creadas por otros autores como Salvador Bartolozzi (1882-1950) e Ismael Smith (1886-1972), entre otros. Su dedicación a la escultura en pequeño formato y su escasa práctica en la obra monumental, además de su manifestada admiración por su compañero, son quizás los motivos de su asociación con Julio Antonio.

\section{El proyecto del monumento a las Américas (Oviedo, 1913-14) y el taller de la calle Montalbán}

El proyecto para el monumento a las Américas en Oviedo fue el primer trabajo realizado conjuntamente por ambos artistas. Miranda, entonces un joven escultor en busca del éxito, fue quien se enteró de la iniciativa ovetense, tomó la decisión de realizar una maqueta y se asoció

\footnotetext{
${ }^{1}$ Entre los estudios más recientes destacan: Santos Torroella, R., Exposición antológica de Julio Antonio, Madrid, 1969; Ricomà Vendrell, X., Julio Antonio, Tarragona, 1983; García de Carpi, L., Julio Antonio. Monumentos y proyectos, Madrid, 1985; GARCíA DE CARPI, L., "El monumento a Chapí de Julio Antonio", Archivo Español de Arte, tomo 58, n. ${ }^{\circ}$ 231, 1985, pp. 251-257; Infiesta, J. M., Julio Antonio, 1988; Ricomà Vendrell, X. y Ricomà Vallhonrat, R., Julio Antonio. Catàleg de dibuix i escultura, Tarragona, 1989; AA.VV., Julio Antonio y su tiempo, Madrid, 1989; AA.VV., El escultor Julio Antonio. Ensayos de aproximación, Tarragona, 1990; SALCEdo MiLIANI, A., Julio Antonio Escultor, 1889-1919, Barcelona, 1997; SALCEDo Miliani, A., "Una nova obra de Julio Antonio al Museo d'Art Modern de Tarragona", en Clau, Tarragona, 2000, pp. 99-101 y AA. VV., Julio Antonio, 1889-1919, catálogo MNCARS, Madrid, 2002.

${ }^{2}$ Miranda ha sido objeto de los siguientes estudios monográficos: Vega Díaz, F., Claves de Sebastián Miranda, escultor, asturiano de pro, Edición privada, Madrid, 1966; Castañón, L., Sebastián Miranda. El Retablo del Mar, Gijón, 1978; Homenaje a Sebastián Miranda [En el centenario de su nacimiento], Oviedo, 1985; GómEZ-SANTOS, M., El tiempo de Sebastián Miranda. Una España insólita, Madrid, 1986 y Álvarez Blanco, M., "La Exposición del Retablo del Mar de Sebastián Miranda en la Biblioteca del Instituto Jovellanos de Gijón en 1933", Ismos. Arte y Música de España y Latinoamérica, n. ${ }^{\circ}$ 1, Universidad de Oviedo, 1999, pp. 15-20. Actualmente, la autora del presente artículo ha defendido su tesis doctoral sobre el artista en la Universidad de Oviedo y dirigida por el profesor Dr. D. Javier Barón Thaidigsmann, a quien agradezco la lectura de este artículo.
} 


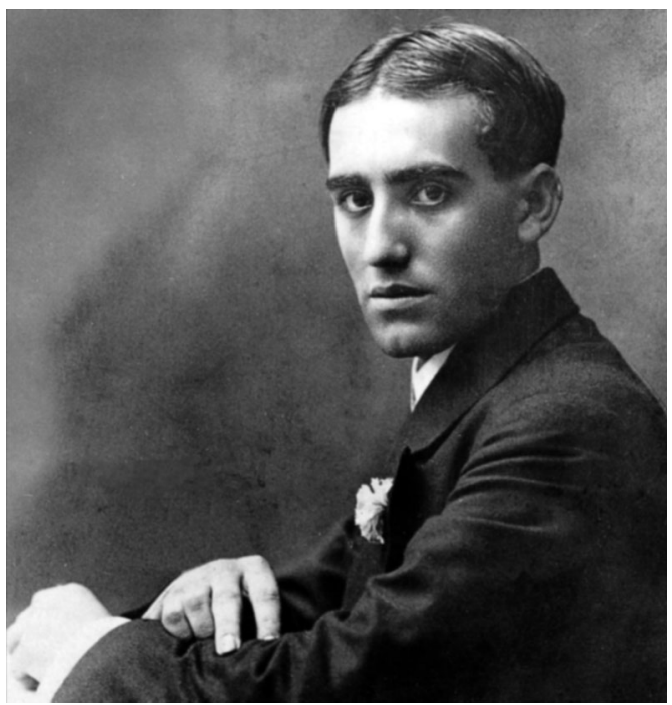

Fig. 1. Julio Antonio. Tarragona, Museo de Arte Moderno. Archivo fotográfico.

Fig. 2. Sebastián Miranda. Archivo familiar.

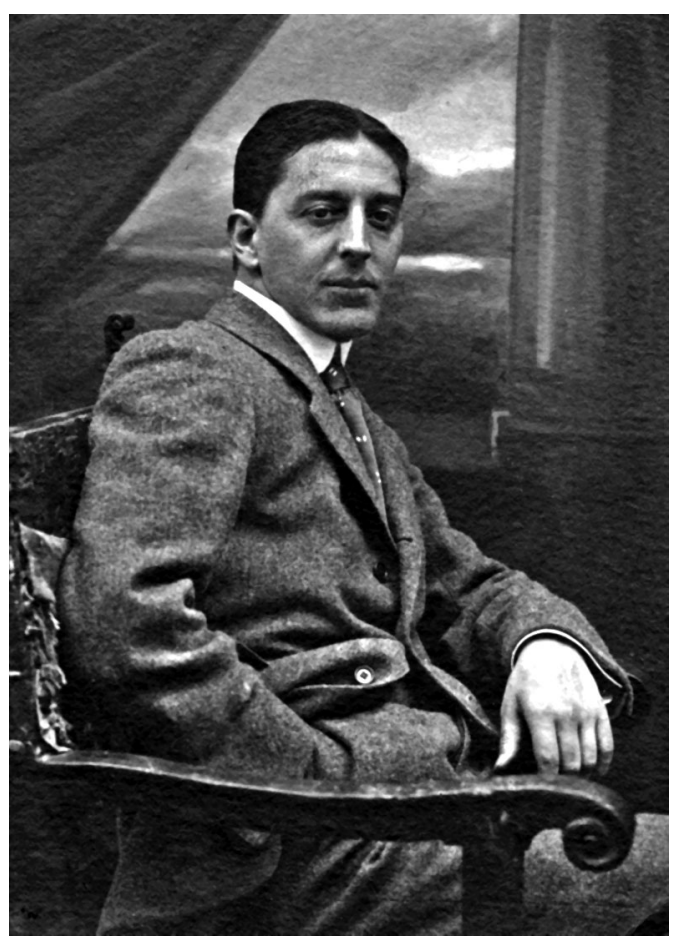

con su compañero Julio Antonio ${ }^{3}$. Así se deduce del siguiente fragmento de una carta dirigida a su madre, María Pérez-Herce Alvargonzález (1855-1933):

Pero vamos al caso concreto. Desde que he sabido el proyecto que tienen en Asturias de levantar un monumento á las Américas, no he tenido un minuto de sosiego. Me uní con Julio Antonio, el escultor que según mi juicio tiene mas talento, y hemos pensado y realizado un proyecto colosal. El boceto que estamos haciendo mide varios metros de altura y lo armaremos dentro de muy pocos días en mi estudio. El presupuesto que hemos hecho es de un millón de pesetas. Nos empeñamos hasta las orejas para llevarlo á cabo. Ahora hazte cargo de las probabilidades que tenemos de triunfar. Primero damos el golpe y la sorpresa puesto que pensaban sacarlo á concurso y nos adelantamos (Un concurso produce desaliento, porque si juzgan de buena fe como el jurado es idiota premian lo mas feo, y si se guían de recomendación y de la intriga es como jugar a la lotería). El boceto, dejando aparte modestia, es realmente colosal, y estamos persuadidos de ello. Tenemos toda la prensa y la intelectualidad de nuestra parte, estamos seguros que están todos dispuestos á favorecernos. Habrá pues una campaña y las personas de más prestigio estarán de nuestra parte. Después de esto esperamos que no haya ningún escultor que se atreva á entablar competencia de ningún género. Me han prometido que la Infanta Isabel ira á mi estudio y podrás imaginarte la campanada que será.

\footnotetext{
${ }^{3}$ Es inexacta, pues, la afirmación de Salcedo de que Miranda había recibido el encargo de una sociedad de asturianos presidida por la Marquesa de Argüelles de realizar un monumento a América. SALCEDO MiLIANI, A., "El escultor Julio Antonio", en Julio Antonio, 1889-1919, ob. cit., p. 34. Conocedor de la iniciativa, se adelantó al concurso, tuvieron éxito, y su proyecto, aunque no se llegó a realizar, fue el elegido.
} 
Apenas duermo de excitación pensando si la suerte que hasta ahora me viene favoreciendo me acompañara ahora para salir de una vez y para toda mi vida, de estas intranquilidades que tengo por la inestabilidad de mi posición. El conde de Agüera forma parte de la comisión y también prometió ayudarme todo lo que el pudiera ${ }^{4}$.

La iniciativa del monumento había sido promovida por la Marquesa de Argüelles ${ }^{5}$, presidenta de la Comisión organizadora, que integraban destacados miembros de la sociedad ovetense. María Josefa Argüelles Díaz, Marquesa de Argüelles († 1947), era la única hija del acaudalado indiano Ramón Argüelles. Cubana de nacimiento, regresó a España con su padre y se casó, en 1883, con Federico Bernaldo de Quirós y Mier, de familia de abolengo y destacado linaje asturiano. María Argüelles protagonizó un importante papel en la sociedad y economía asturianas, así como en la española, y contó con residencia en Madrid y un trato frecuente con la familia real. Interesada por el arte, fue una notable impulsora de éste y de otras actividades de carácter cultural y social, como lo fue en general el colectivo indiano en la sociedad asturiana, promotor de la economía, la industria, la construcción, la educación y el arte de la región.

Es interesante destacar que Sebastián Miranda, natural de Oviedo, conocía y había tratado con los miembros de la Comisión, lo que podría influir en una decisión a su favor, en un doble favoritismo: personal y local, por tratarse de un artista de la región. Miembro de una destacada familia burguesa asturiana, Miranda mantuvo una estrecha relación con los Marqueses de Argüelles (a quienes había retratado en la década anterior) y con el conde de Agüera, padre de Julián Cañedo, íntimo amigo de Miranda y también de Julio Antonio.

Miranda y Julio Antonio se habían conocido hacia finales de 1910 o principios de 1911, época en la que los dos se habían instalado en Madrid en busca del éxito profesional: Julio Antonio recién llegado de Almadén y Sebastián Miranda de Roma. Según contó el artista asturiano en sus anecdóticas conferencias ${ }^{6}$, cuando iba a solicitar una pensión para la Real Academia de Bellas Artes de Roma, su amigo Ramón Pérez de Ayala (1880-1962) le informó de que otro escultor, que compartía estudio con Miquel Viladrich (1880-1956) en la calle de Villanueva, tenía la misma intención ${ }^{7}$. Al ir a visitarle, instigado por Pérez de Ayala, descubrió un artista genial, al que no dejaría desde entonces de admirar. Esto le llevó a tomar la decisión de no presentarse a la pensión, en favor del artista tarraconense. Julio Antonio tampoco la solicitó.

La carta aquí extractada, fechada en noviembre, nos permite fijar el inicio de esta colaboración en el último trimestre de $1913^{8}$. Al menos hasta enero de 1914, momento en que fue presentada la maqueta, o febrero, cuando recibió la visita de la infanta Isabel ${ }^{9}$, ambos escultores compartieron trabajo y taller. Instalados en el estudio del artista asturiano, en el sexto piso de la calle Montalbán número 17, el taller se constituyó como un núcleo de debate, bohemio e intelectual, continuación de las reuniones de los cafés. Allí recibían la visita de numerosos amigos e intelectuales, y mientras ideaban el monumento, se discutía a su alrededor sobre varios temas, entre ellos el de los toros. Según una crónica de la época, el estudio compartido por Miranda y Julio Antonio era visitado casi todas las tardes por Valle Inclán,

${ }^{4}$ Carta de Sebastián Miranda a María Pérez-Herce, El Milanillo, 15-11-(1913). Archivo familia del artista.

5 "Madrid Asturias", en Asturias. Revista Ilustrada del Centro Asturiano, Madrid, n. ${ }^{\circ} 343$, febrero 1914, p. 28. Probablemente el anuncio de dicha iniciativa fue publicado en la prensa local, aunque no se ha localizado ninguna referencia al respecto.

${ }^{6}$ Miranda, S., "El doctor Marañón”, borrador de la conferencia "El Doctor Marañón que yo conocí", conservado en la Fundación Gregorio Marañón, Madrid.

7 Gómez-Santos, M., El tiempo de..., ob. cit., p. 53.

${ }^{8}$ Salcedo Miliani, A., Julio Antonio..., ob. cit., p. 135, afirma que compartían taller en 1916, momento en que realizarían este trabajo.

9 "Dos artistas asturianos", El Noroeste, Gijón, 10-2-1914, p. 4. 
Pérez de Ayala, Enrique de Mesa, Luis de Tapia y Julián Cañedo. Uno de los temas favoritos era el de los toros. Disfrutaban montando corridas en el estudio, en las que hacía de toro un gitano muchacho llamado Montaño, que también cumplia las funciones de modelo. Llegaron incluso a organizar un evento taurino anunciado como festival aristocrático (...) ${ }^{10}$. También se discutía sobre literatura, arte y danza, debido a la presencia de escritores y artistas ligados a otras corrientes de la época: Al taller con espíritu de alta crítica digna de seguir sus sabias enseñanzas, acuden a diario maestros de la literatura y arte españoles: Valle-Inclán, el manco ilustre que, a semejanza de otro manco excelso, riñe, batallas formidables con la incultura ambiente en favor de las Letras de su Patria; Pérez de Ayala y Luis Bello, Romero de Torres y Anselmo Miguel Nieto; Jacinto Benavente, el más insigne dramaturgo de la Europa actual, según textos franceses; Tapia, Enrique de Mesa, Candamo.../ Allí en Areópago intelectual, discuten las últimas producciones literarias, sientan reglas de arte; de vez en vez, sobre los barros modelados corrigen líneas, orientan actitudes. Y un día la Argentina, esa bailarina maestra que hizo de unos palillos andaluces cajas de dulces armonías y educó el ritmo de su cuerpo flexible en danzas en que Grecia y España se fundían, y otra la Imperio, la bailaora genial que entre reflejos cálidos de sus carnes de bronce hace al baile gitano arte sublime, y otro la Goya gentil riente y luminosa, dan pintorescas notas de su arte entre aquellos artistas $y$ presiden, entre flores y estatuas, festejos en su honor ${ }^{11}$.

En este ambiente nació el proyecto para el Monumento a las Américas. Pese a la buena acogida que tuvo el boceto y a la aceptación de la Comisión Organizadora del Monumento, no se llegó a realizar, probablemente por motivos económicos. Conocemos su concepción gracias a varias reproducciones publicadas en la prensa gráfica de la época (fig. 3), a un apunte del remate del conjunto (fig. 5), a un boceto en bronce del grupo de coronación del monumento (fig. 4) y a los comentarios de diversos críticos publicados en los rotativos del momento ${ }^{12}$. Como apuntaba Miranda en carta a su madre, la intelectualidad estuvo de su parte.

El Monumento a las Américas o a América se pensaba erigir como homenaje al continente americano y como muestra de gratitud a los paises donde hallaron la ansiada recompensa y esfuerzo ${ }^{13}$, voluntad que refleja una iniciativa indiana. Fue ideado con proporciones colosales ( 30 metros de altura por 18 de base $^{14}$ ) y con destino a una plaza entonces sin fijar en la ciudad ovetense $^{15}$. Estas dimensiones justificaban casi con seguridad el, para la época, desorbitado presupuesto de un millón de pesetas.

El monumento se componía de una pirámide cuadrangular truncada con cuatro contrafuertes trapezoidales en las aristas, elevada sobre un pedestal rectangular y tres grandes escalones de sillares ciclópeos. Esta estructura arquitectónica, que predominaba en el conjunto, iba a estar

10 Villa, A. de la, "Cómo fue la vida de Julio Antonio", La Libertad, Madrid, 14-2-1920.

11 Gillis, F., "Nuestros artistas. El monumento a América", El Mundo, recorte de prensa sin fecha del archivo de Sebastián Miranda, Colección Virginia Rebollar, Murcia.

12 Estos artículos, indispensables para analizar la obra, son: ABriL, M. "Un monumento de Julio Antonio y Sebastián Miranda", Nuevo Mundo, Madrid, 15-1-1914; AlCÁntARA, F., "Notas de Arte. Los asturianos y América. Monumento de los escultores Julio Antonio y Sebastián Miranda", El Imparcial, Madrid, 11-1-1914, p. 3; LAGO, S. (seudónimo de José Francés), "Bellas Artes. Pintores y escultores”, La Esfera, Madrid, 7-3-1914; PÉrEZ DE AYALA, R., "Julio Antonio y Sebastián Miranda", Gran Mundo, Madrid, 15-3-1914, pp. 10-11 y Gillis, F., "Nuestros...", art. cit. Salcedo hace también un interesante, aunque incompleto, análisis en: Julio Antonio Escultor, ... ob. cit, pp. 134-137 y "Una nova obra...", art. cit, pp. 99-101, donde presenta el boceto escultórico.

13 “Actualidades. Informaciones gráficas de la Semana", Blanco y Negro, Madrid, 25-1-1914.

14 Alcántara, F., "Notas de Arte...", art. cit.

${ }^{15}$ Existe en la actualidad una Plaza de América en Oviedo, dotada de una monumental fuente. Se plantea pues la posibilidad de que este fuera el emplazamiento original, no muy alejado del ensanche urbano ocupado por las mansiones indianas, aunque no se ha localizado ninguna prueba documental al respecto.

AEA, LXXXI, 321, ENERO-MARZO 2008, 49-66, ISSN: 0004-0428 


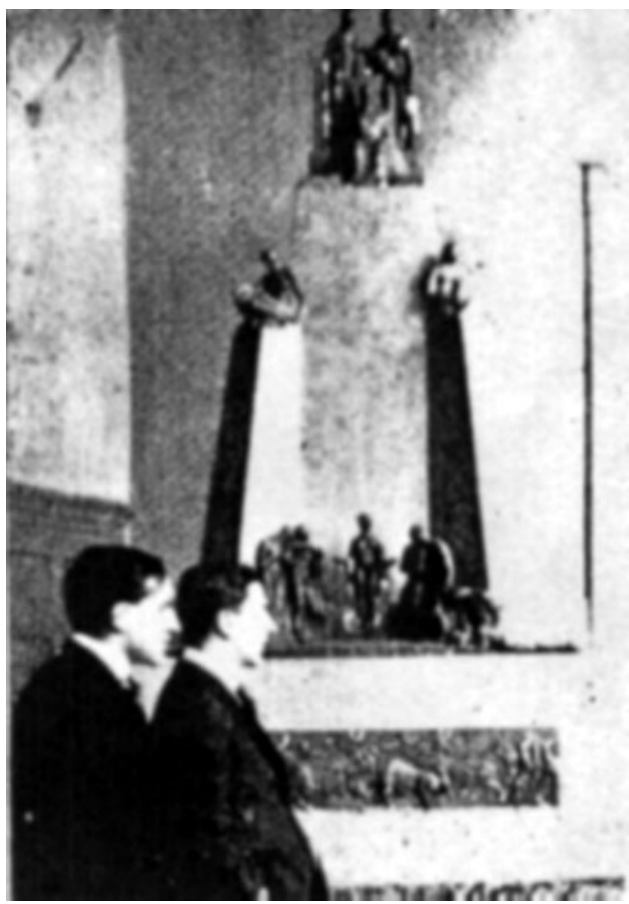

Fig. 3. Julio Antonio y Sebastián Miranda presentando la maqueta del Monumento a las Américas.

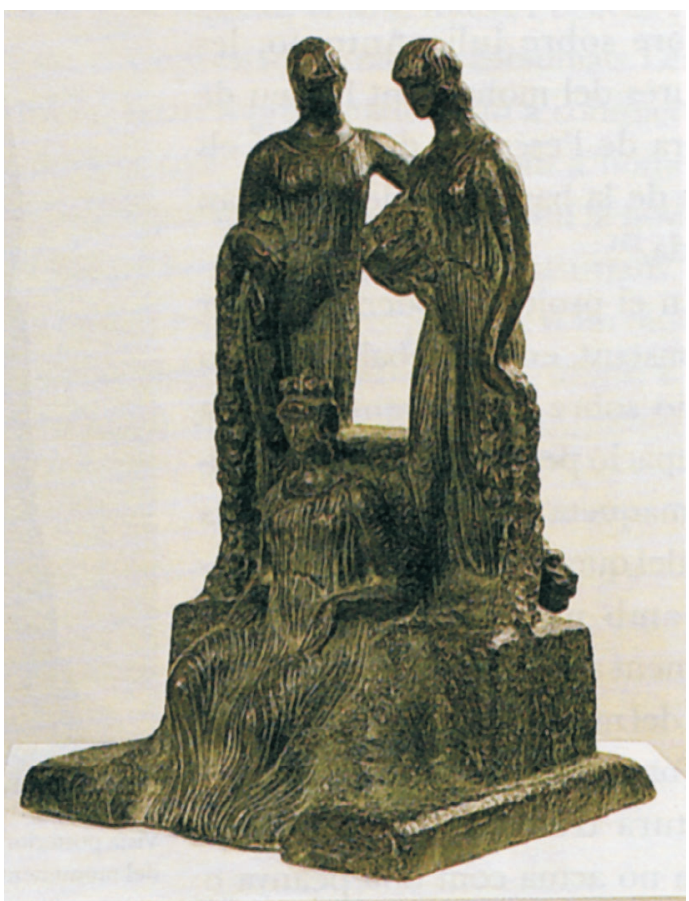

Fig. 4. Boceto de la coronación del Monumento a las Américas. Madrid, Colección particular.

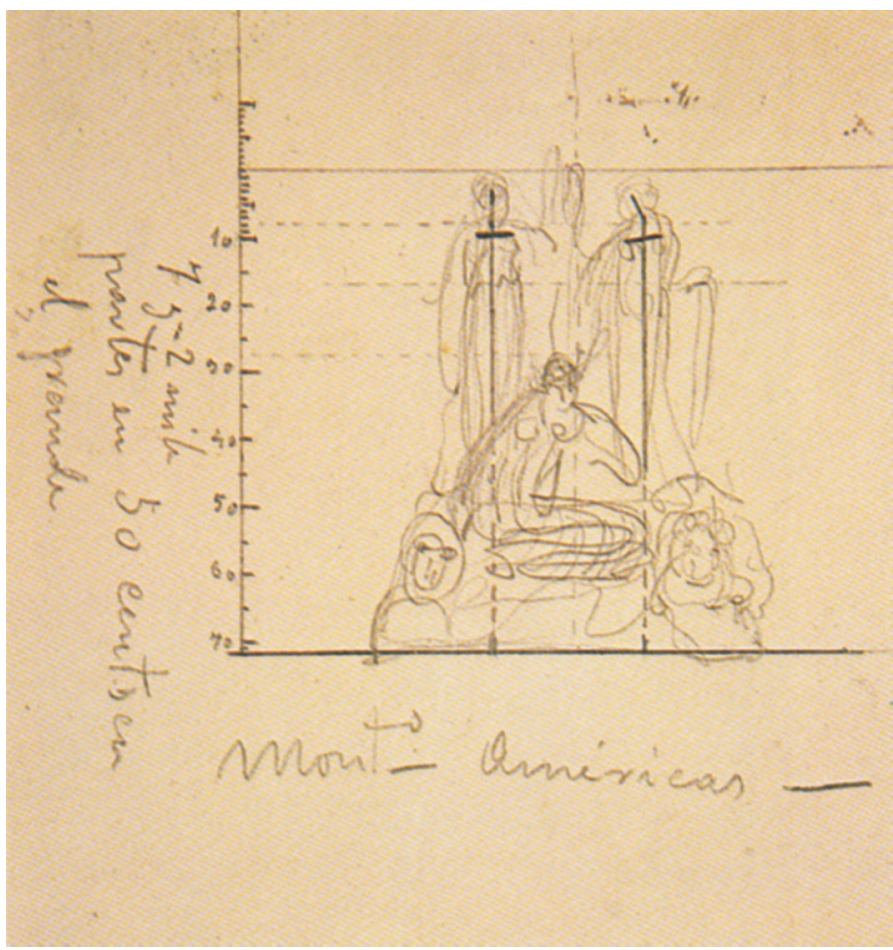

Fig. 5. Boceto del Monumento a las Américas. Tarragona, Museo de Arte Moderno. 
realizada en piedra. Ramón Pérez de Ayala elogiaba la elección de la pirámide como eje de la composición por sus antecedentes clásicos: Ya Platón sostenía que, en la vertical y en el triángulo, se encierran maravillosamente las normas madres de toda belleza. Siglos después, Leonardo procuraba agrupar y componer siempre sus figuras dentro de un esquema triangular (... ${ }^{16}$. No hay que olvidar que Ramón Pérez de Ayala defendía en sus teorías estéticas la recuperación del clasicismo en la escultura contemporánea y que fue un profundo admirador de la antigüedad griega y del Renacimiento italiano.

En cuanto a la parte escultórica, integrada por varios grupos en bronce situados a distintas alturas, acorde con la disposición piramidal, tenía su temática en torno al encuentro entre ambos continentes. Gracias a los comentarios de Manuel Abril, Francisco Alcántara, Fernando Gillis, Silvio Lago (seudónimo de José Francés) y Ramón Pérez de Ayala conocemos cada uno de estos conjuntos y su significado.

A modo de coronación de la pirámide se situaba el primer grupo, compuesto de tres figuras femeninas, una central sentada y dos laterales, de pie. Otros dos grupos de una media docena de figuras se situaban en ambos frentes del monumento, sobre la base cuadrangular y anexos a la pirámide truncada. Cuatro figuras sobre cada uno de los contrafuertes y un friso de 2,5 metros de altura por 10 de longitud desarrollado a lo largo del basamento completaban el programa figurativo.

Las figuras de coronación representaban a las dos Américas (las mujeres de pie portando guirnaldas y frutos) y a España (la matrona sentada). Como se puede observar en el boceto (fig. 4) las mujeres de pie se disponían sobre una estructura en forma de podio rodeando a la matrona sentada y portando guirnaldas. Se plasmaba así la idea de dominio de Europa sobre América, al estar las dos alegorías de América, del Norte y del Sur, dispuestas a modo de protección y veneración de la sedente y coronada Europa.

Sobre cada uno de los contrafuertes se disponían cuatro hombres desnudos símbolo de los cuatro ríos más importantes de América: Plata, Mississippi, Amazonas y Paraná.

Otras alegorías encontramos en los dos grupos inferiores. En el frontal se situaba el grupo del "Descubrimiento o la Invención de América", compuesto por una figura central femenina desnuda. De pie, y representando a América con un manto dorado entre sus brazos, estaba rodeada de otras masculinas también desnudas en diferentes actitudes. Dispuestas en composición triangular, al modo de un frontón griego, representaban la curiosidad de los descubridores ante el nuevo continente. En la parte posterior se situaba el grupo de la América actual, industriosa y rica, que se ofrecía a la contemplación de las gentes, con una disposición semejante al grupo frontal.

Por último, el relieve del basamento, tratado con un naturalismo realista que contrastaba con el idealismo de los grupos anteriores, recogía diversos aspectos de la ganadería y la agricultura americana, fuentes de riqueza del continente. En el frente se situaba el ganado vacuno; a la derecha, el ganado caballar; en el posterior el ganado lanar y a la izquierda la industria agrícola.

Las esculturas, de bronce, irían acompañadas por matices de oro: Doradas a fuego irán las figuras que rematan el monumento y las de los cuatro ríos; en el grupo de abajo estrías de oro; en el paño de la figura central, algunos toques en guirnaldas y en hojas -muy sobriamente-, realzarán la noble severidad del bronce, sobre la piedra, que las aguas y el tiempo patinarán con musgos y ámbar de sol $l^{17}$.

Éste fue el resultado final de varios meses de ideas y trabajo, de los que debieron nacer numerosos apuntes y bocetos. Uno de ellos es el conservado en el Museo de Arte Moderno de Tarragona (fig. 5), fragmentario, de la coronación del monumento. En él se aprecian ya las dos figuras femeninas de pie, rodeando a otra, en este caso arrodillada, con dos leones a sus lados. Fernando

\footnotetext{
16 PÉrez de Ayala, R., “Julio Antonio...”, art. cit.
}

17 ABril, M., "Un monumento...", art. cit. 
Gillis habla de los orígenes de este grupo, del que este estudio sería un estadio posterior, y que culminaría en la maqueta antes comentada, que redujo la presencia de esfinges y leones a un león esculpido caminando en la parte posterior del podio de coronación, probablemente símbolo del poder. De su texto se deduce también cómo la creación del proyecto fue fruto de un trabajo común entre ambos escultores ${ }^{18}$ :

Julio Antonio debió pensar su proyecto en uno de sus sueños egipcios: dos de las pirámides del Cairo, una sobre la otra, y en el remate, tragándose con sus ojos vacíos tierras y tierras, hasta llegar a América, la esfinge símbolo con sus garras de león, del león de Iberia. Y todo ello frente al mar, sobre la gran extensión de terreno que se necesitaría para que fuese visto el monumento desde el propio Nueva York.

Miranda, pasado el asombro del momento, debió empezar a reducir los bloques pétreos. A más, la esfinge, de nariz rota y barbilla deshecha, no iba bien; en su lugar debía elevarse España, no la matrona augusta, rígida, con etiqueta cortesana, sino la majestad y la grandeza hechas mujer de bellas líneas, resplandeciendo en ella la severidad griega, fuerza viril en cuerpo femenino, carnes llenas y fuertes músculos, bajo flexibles morbideces... ${ }^{19}$

La importancia del marco arquitectónico, integrado con las esculturas, y la existencia de cuatro contrafuertes rematando las esquinas del mismo, son dos elementos empleados por Julio Antonio en otros proyectos de monumentos. Aparecen, por ejemplo, en un boceto para el Monumento a Saavedra, de 1913 (Museo de Arte Moderno de Tarragona, NIG 690) y en sus bocetos para el monumento a Cervantes, de 1915 (Museo de Arte Moderno de Tarragona, NIG 609 y 693), aunque en el caso del monumento a las Américas la integración entre arquitectura y escultura es mucho mayor.

Por otra parte, destaca también el idealismo y la alegoría que predomina en toda la concepción escultórica. En un intento de recuperación del arte clásico, las figuras se llenan de referencias a modelos griegos arcaicos (vestimentas, pliegues de la ropa, peinados) y renacentistas (desnudos masculinos casi miguelangelianos), al tiempo que se emplean recursos iconográficos clasicistas como el tema de los cuatro ríos, o las flores, frutas y guirnaldas. En el plano formal, ya hemos aludido a la referencia de la disposición piramidal de la estructura y de frontón de los grupos inferiores.

Todos los críticos coincidieron en señalar la modernidad a través del clasicismo del monumento, y su pureza de líneas. En especial Silvio Lago y Ramón Pérez de Ayala, quienes abogaban por una renovación de las artes plásticas españolas a través de la recuperación clásica griega y romana. Julio Antonio y este proyecto realizado con Miranda, son dos muestras de este intento de renovación elogiadas por los pensadores del momento.

${ }^{18}$ Salcedo había afirmado que el autor principal y director del diseño fue Julio Antonio, mientras Miranda tendría un papel secundario, aceptando sus ideas y modelando los relieves de animales y agricultura (SALCEDo Miliani, A., Julio Antonio..., ob. cit., p. 137). Aunque es cierto que las directrices generales del monumento son más deudoras de la estética de Julio Antonio, apreciamos en el texto extractado la total colaboración.

${ }^{19}$ Gillis, F., "Nuestros...", art. cit. Fernando Gillis era, además, amigo de los artistas, y visita frecuente en su taller.

AEA, LXXXI, 321, ENERO-MARZO 2008, 49-66, ISSN: 0004-0428 


\section{El monumento a Manuel Camo (Huesca, 1915)}

El monumento a Manuel Camo fue el segundo proyecto conjunto de Julio Antonio y Sebastián Miranda. Atribuido tradicionalmente a Julio Antonio, ha sido objeto de una serie de errores historiográficos, continuados sin revisión hasta nuestros días ${ }^{20}$.

Manuel Camo Nogués (1841-1911) fue un farmacéutico y político natural de Huesca, cuyo nombre aparece siempre asociado al caciquismo. Fue uno de los políticos oscenses más destacados de la Restauración. Hasta 1898 perteneció al Partido Republicano Posibilista, cuya máxima figura era Emilio Castelar. Para él consiguió la representación del distrito electoral de Huesca desde 1881 hasta 1898. En ese año, sus diferentes posturas sobre la guerra de Cuba y la política colonial hicieron que Camo se orientara hacia el liberalismo de Sagasta. Camo fundó su propio periódico, El Diario de Huesca, desde el que imponía sus ideas, y ocupó los cargos de Diputado a Cortes por Huesca, Senador vitalicio por designación Real y Vicepresidente de la Diputación Provincial $^{21}$.

Su muerte, acaecida el 26 de diciembre de 1911, determinó la creación de una comisión para elevar un monumento en su honor. El Directorio que le había sucedido en la dirección del partido fue el promotor del proyecto. En enero de 1912 se nombró una comisión Ejecutiva integrada por íntimos amigos de Camo que se encargó de llevar a término la idea e impulsar la suscripción popular $^{22}$. Según María José Calvo, las aportaciones a esta suscripción provinieron de toda la provincia, de Argentina y de Barcelona, recaudándose un total de veintiocho mil trescientas cuarenta y ocho pesetas.

La responsabilidad de elegir quién iba a ejecutar el monumento recayó sobre Justo Martínez, amigo del cacique y editor en Madrid del Diario de Huesca, quien optó por Sebastián Miranda. Según recordaba el artista asturiano, el encargo le fue concedido por su amistad con el torero Juan Belmonte (1892-1962). El editor oscense, profundo admirador del torero, consideró que le halagaría concediéndole un encargo a uno de sus mejores amigos ${ }^{23}$. La adjudicación tuvo lugar

${ }^{20}$ Pío Baroja, Miguel de Unamuno y Ramón del Valle-Inclán citan este monumento como obra de Julio Antonio y se refieren al personaje como modelo de caciquismo: BAROJA, P., "Las horas solitarias", p. 269; "Tres generaciones", p. 573 y "Divagaciones apasionadas", p. 497, los tres en Obras Completas, tomo V, Madrid, 1946-1951; VALLE-InCLÁn, R. M., Luces de bohemia, Madrid, 1980; y Unamuno, M. de, "Costa y Camo", El Debate, Barcelona, 1-12-1916, p. 1, artículo publicado en respuesta a Aznar Navarro, F., "Significación de un monumento", Correspondencia de España, Madrid, 27-11-1916, p. 3. Es curioso señalar que, a pesar de la amistad de Baroja y Valle-Inclán con Miranda, citaran el monumento únicamente como obra de Julio Antonio, lo cual sirvió de base al mantenimiento posterior de esta atribución. Así, GARCÍA DE CARPI, L., Julio Antonio..., ob. cit., p.13 y "Luces y sombras de una actuación”, en Julio Antonio, catálogo MNCARS, Madrid, 2002, pp. 69-70; y SAlCEDo Miliani, A. Julio Antonio..., ob. cit., p. 119-120 atribuyen este monumento únicamente a la intervención de Julio Antonio. Indican, además, como estaba dedicado a José, y no Manuel, Camo. José Camo, también farmacéutico, fue el padre del político (Gran Enciclopedia Aragonesa 2000, Zaragoza, 2000, tomo 4, p. 936). Por otra parte, Sebastián Miranda ha hablado en sus memorias de un monumento a un político realizado al alimón con Julio Antonio en un pueblo de Extremadura (MirAndA, S., "Al alimón con el escultor Julio Antonio", ABC, Madrid, 6-1-1963; recogido en Recuerdos y añoranzas (Mi vida y mis amigos), Madrid, 1973, pp. 4147). Sin embargo, una serie de coincidencias que se desglosarán en el texto (los tres bocetos, el barbero, el caciquismo, la dirección de un periódico, etc.) me llevan a pensar que se trata del mismo monumento, situado en Aragón en lugar de en Extremadura, y localizado erróneamente en la memoria de Miranda, máxime cuando no se ha localizado ningún dato sobre el supuesto monumento en Extremadura.

${ }^{21}$ Para más información sobre esta figura véase García Mongay, F., Manuel Camo Nogués. El cacique de Huesca (Apuntes para una biografia), Cuadernos del Alto Aragón, Zaragoza, 1985.

22 Calvo Salillas, M. J., Arte y sociedad: actuaciones urbanisticas en Huesca, 1833-1936, Huesca, 1990, p. 110. También Calvo Salillas, M. J. y Alvira Banzo, F., El Círculo Oscense. Cien años de historia, Huesca, 2005.

${ }^{23}$ Miranda, S., "Al alimón...”, art. cit. María José Calvo facilita el nombre del encargado de la comisión. Es probable que Miranda confunda su nombre, que él atribuye a Venancio Cabal, pues éste no aparece en la lista de la 
en marzo de $1915^{24}$. Según Miranda, Julio Antonio, necesitado de dinero, amigo y ya antes colaborador suyo, le ofreció su ayuda:

El caso llegó en seguida a oídos de mi amigo, el extraordinario escultor Julio Antonio, a quien yo admiraba y quería mucho. Me brindó su ayuda, que acepté jubiloso, y convinimos repartir a medias las ganancias. Como prueba de gratitud se ofreció a hacer el boceto que yo acepté gozosísimo por ser una garantía de que la obra iba a tener un gran éxito. En efecto, pocos días después me llamó para ver lo que había hecho y quedé maravillado al comprobar el ingenio y la gracia con que lo había resuelto.

Trasladamos el boceto a mi taller y avisamos a la comisión que previamente se había formado a base de lo que llaman fuerzas vivas ${ }^{25}$.

De estas palabras deducimos cómo ambos escultores trabajaban de nuevo de manera independiente, cada uno en su taller, y se unieron en esta ocasión por motivos económicos. Julio Antonio fue el autor del primer boceto del monumento, que fue rechazado por la comisión organizadora. A éste siguió un segundo modelo, también obra del tarraconense e igualmente rechazado. El tercer y último diseño fue realizado por Sebastián Miranda, quien lo describió como una verdadera birria $^{26}$. Sin embargo, su proyecto fue aceptado en mayo de 1915, momento en que acudió a Huesca para presentarlo en compañía de Ramón del Valle-Inclán y Ramón Pérez de Ayala ${ }^{27}$. Se firmó un contrato y el monumento, destinado a ocupar la plaza del Teatro de Huesca ${ }^{28}$, fue realizado según el modelo de Miranda en el taller que Julio Antonio tenía en la Fundición Codina. El 26 de noviembre de 1916, y tras un largo proceso de cambios en la idea primigenia e intrigas, era solemnemente inaugurado (fig. 9) ${ }^{29}$.

En el Museo de Arte Moderno de Tarragona se conservan dos bocetos diferentes de este monumento, que corresponden casi con seguridad a los dos diseños de Julio Antonio antes aludidos (figs. 6 y 7). El primero de ellos (NIG 689) muestra, con un trazado muy abocetado, un alto y sobrio pedestal rectangular con cuatro aleros sobresaliendo en la base. Sobre el pedestal, y de pie, una figura femenina alegórica de corte clásico y portadora de una guirnalda, muy similar a las que ya se han comentado en el Monumento a las Américas. En la parte anterior del basamento, otra mujer arrodillada. En el frontal del pedestal, un espacio reservado

comisión organizadora publicada con motivo de su inauguración, ENA, "Huesca al día. Homenaje a la memoria de Camo", Heraldo de Aragón, Zaragoza, 27-11-1916, p. 2, al igual que son erróneas las fechas (1914) y la localización (Extremadura) que él nos proporciona.

24 Ena, "Huesca al día. Un monumento", Heraldo de Aragón, Zaragoza, 18-3-1915, p. 1. Calvo Salillas, M. J., Arte y sociedad..., ob. cit., p. 110 afirma que el proyecto les es encargado en enero de 1913, al tiempo que atribuye la escultura a Julio Antonio. Desconozco la procedencia de este dato que, al contrastarlo con la noticia periodística que afirma cómo se le adjudica la obra en marzo de 1915 y cómo es aceptado el proyecto definitivo en junio de 1915, parece sería demasiado temprana. Por ello considero que los proyectos del monumento serían de estos meses centrales de 1915 y posteriores al proyecto del Monumento a América que, según parece indicar la carta aquí extractada, fue su primera colaboración.

${ }^{25}$ Miranda, S., “Al alimón...”, art. cit.

${ }^{26}$ Ibidem.

27 ENA, "Huesca al día. Un monumento", Heraldo de Aragón, Zaragoza, 28-5-1915, p. 1. Habría que retrasar, pues, la fecha propuesta por Salcedo hasta 1915, Salcedo Miliani, A., Julio Antonio..., ob. cit., p. 119.

28 EnA, "Huesca al día. La estatua de Camo", Heraldo de Aragón, Zaragoza, 4-6-1916, p.2. Posteriormente Plaza de Camo y Plaza de la Constitución, ahora de la Inmaculada, aunque inicialmente proyectado para ser construido en Loarre. La obra obtuvo licencia de emplazamiento el 1 de junio de 1916, Archivo Municipal de Huesca, expediente 1.616.

${ }^{29}$ EnA, "Huesca al día. Homenaje...", art. cit. Tanto Miranda como los archivos de la Fundición Codina, Codina Hermanos. Libro de Obras realizadas, 1915-1929, libro 2, escultura fundida entre junio-julio de 1916, confirman el dato de su fundición y atribución a Julio Antonio, aunque la escultura pueda asignarse, por sus características, a Miranda. 
para un relieve y una inscripción, dedicadas casi con seguridad a la efigie y ponderación del homenajeado.

Este primer boceto, rechazado por poco alusivo, dio paso a un segundo (NIG 700). Partiendo del anterior, Julio Antonio diseñó un monumento consistente en un desarrollado cuerpo rectangular con columnillas en las esquinas, sobre basamento rectangular y dos escalones. Sobre el pedestal, de nuevo, una figura alegórica de una mujer portando guirnaldas, como símbolo de la eternidad y la fama del cacique. En el cuerpo rectangular, y rodeada de guirnaldas, el relieve clásico y de perfil de una mujer, de sentido simbólico desconocido.

En ambos proyectos primaba el sentido intemporal del monumento y se evitaba la representación realista del homenajeado, aludiéndose a él mediante referencias alegóricas. Julio Antonio era muy propenso a este tipo de representaciones en sus proyectos monumentales. Así lo apreciamos en este caso, y también en otros, como por ejemplo en el monumento a Saavedra, que en sus primeros estudios incluían una serie de figuras de esta índole. En éste, como en el de Camo, tuvo que acabar incluyendo sólo la figura del laureado.

El segundo proyecto, similar al anterior, fue la base del diseño de Miranda. El escultor asturiano conservó la estructura arquitectónica del pedestal de sillar revestido de placas de mármol ideado por Julio Antonio. De él eliminó toda muestra de la alegoría, el simbolismo y el clasicismo julioantonianos hasta reducirlo a una concepción totalmente académica. Sustituyó así la figura de coronación por una realista representación sedente de Manuel Camo, y el relieve por un escudo de la villa. Con ello se adaptó, aunque a disgusto de los autores, al gusto decimonónico que seguía imperando en la erección de monumentos.

Aunque atribuida durante años a Julio Antonio, la figura del cacique se relaciona más con la producción de Miranda (fig. 10). De carácter realista, con poco interés por el detalle de la vestimenta y un acabado del bronce muy pulido, recuerda a otros retratos de esos años del escultor asturiano, como el busto del monumento a Jerónimo Ibrán (dos versiones, en Mieres y Madrid), de 1912, o el retrato de Egidio Gavito Bustamante en su monumento en Poo de Llanes (Asturias), de 1911. En este último, y como en el caso de Camo, se representa al también político sobre un alto pedestal y sentado, aunque en el caso de Gavito está ligeramente inclinado hacia un lado, dando una mayor sensación de movimiento ausente en el monumento oscense. Por otra parte, esta estática tipología de retrato sedente alude a la profesión del retratado, entre papeles y oficinas, al modo de los escritores y artistas.

Además de ceder en sus pretensiones artísticas, los escultores tuvieron otros problemas en la realización de este monumento. En primer lugar, se incumplió el contrato, recibiendo el dinero en esporádicos y exiguos plazos y descontando de sus honorarios el coste de la verja y el jardincillo que lo rodeaban en su ubicación definitiva ${ }^{30}$. Por otra parte, la comisión contrató al que fuera barbero del cacique para que supervisara la realización del retrato. Con sus observaciones, dificultó de manera exasperante la elaboración del mismo, hasta que Miranda, en una muestra de picaresca pareja a la demostrada por el barbero, compró su aprobación a cambio de poder terminar la obra ${ }^{31}$.

El monumento tuvo una desafortunada existencia (fig. 8). Dada la fama del político el 14 de abril de 1931 la figura de Camo fue derribada y arrastrada por las calles de la ciudad, siendo definitivamente desmontado y desalojado de su emplazamiento en marzo de 1936. Destruido el pedestal, la figura fue cortada. En la actualidad sólo se conserva un busto en el Círculo Oscense, asociación promovida por el cacique ${ }^{32}$.

\footnotetext{
${ }^{30}$ Miranda, S., "Al alimón...”, art. cit.

31 Ibidem. La historia del barbero es también comentada por Margarita Nelken en "Julio Antonio", Museum, Barcelona, 1919, vol. 5, p. 418.

32 Agradezco a la historiadora Laura Oliván su colaboración al orientarme sobre la actual localización y el devenir del monumento.
}

AEA, LXXXI, 321, ENERO-MARZO 2008, 49-66, ISSN: 0004-0428 


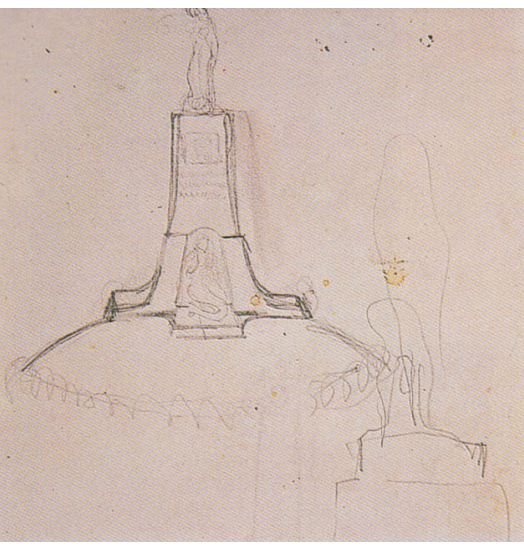

Fig. 6. Primer boceto del Monumento a Camo (1915). Tarragona, Museo de Arte Moderno.

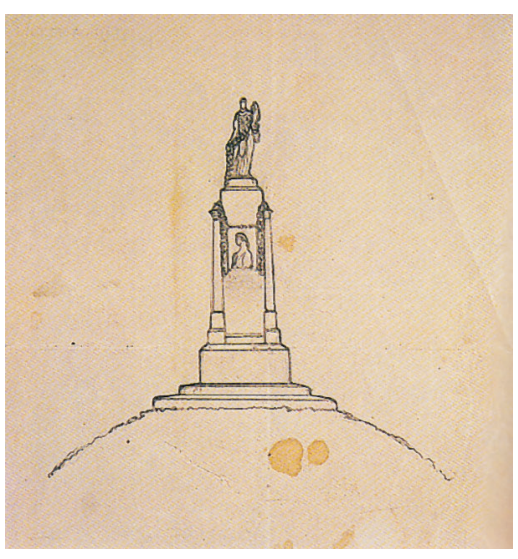

Fig. 7. Segundo boceto del Monumento a Camo. Tarragona, Museo de Arte Moderno.

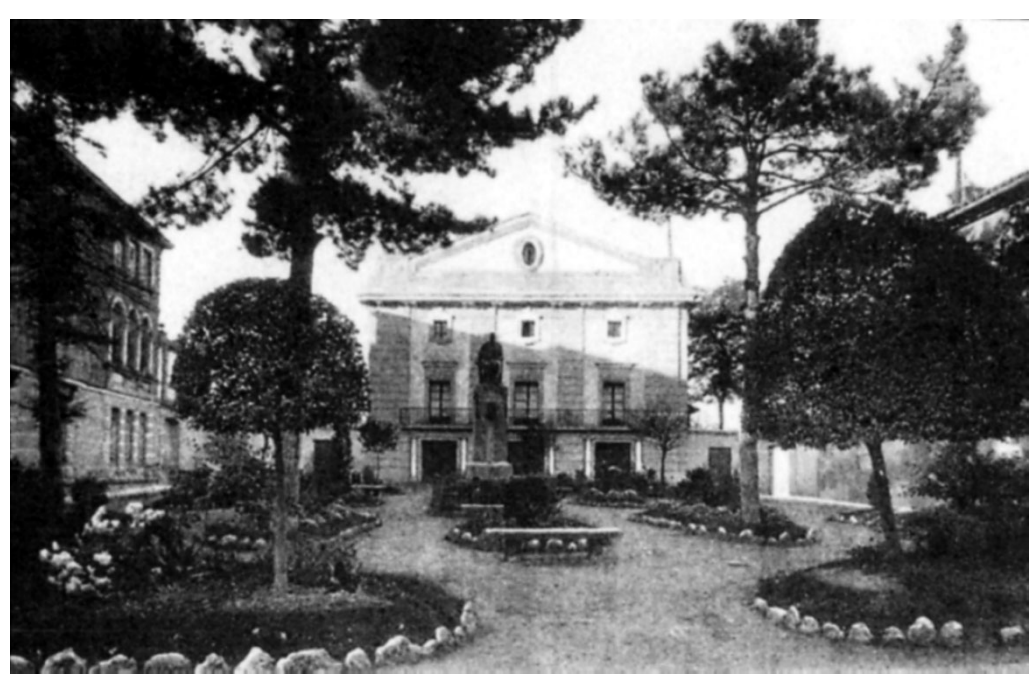

Fig. 8. Huesca. Teatro principal y plaza de la Constitución, con el monumento a Camo. L. Roisin, fotógrafo.

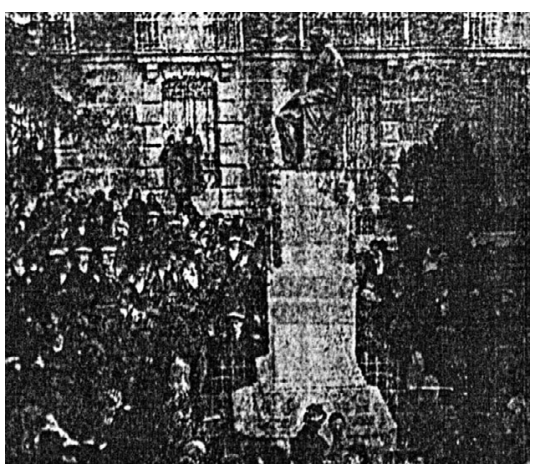

Fig. 9. Inauguración del Monumento a Camo (noviembre de 1916). Fotografía E. Capella. Cliché Heraldo.

Fig. 10. Busto de Manuel Camo en su estado actual. Huesca, Círculo Oscense.

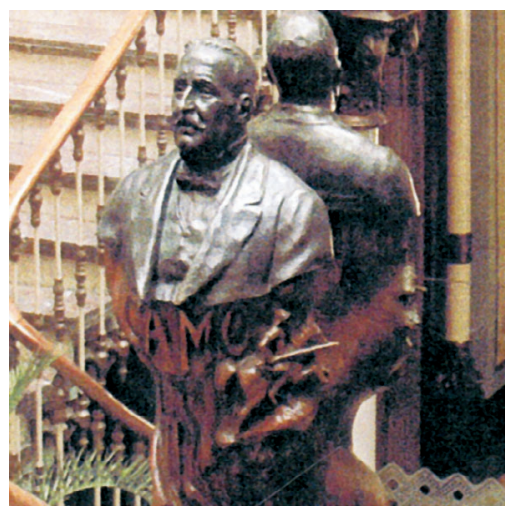

AEA, LXXXI, 321, ENERO-MARZO 2008, 49-66, ISSN: 0004-0428 


\section{El proyecto para el monumento a Pedro Menéndez de Avilés (Avilés, Asturias, 1916)}

Una de las figuras clave en la historia de la localidad asturiana de Avilés fue la de Pedro Menéndez. Destacado marino nacido en Avilés en 1519, falleció en Santander en 1574. En un principio, se dedicó al corso contra los piratas que navegaban por las costas del Cantábrico, hasta que Felipe II lo puso a su servicio. Fue Capitán General de la Flota de Indias, luchó en Flandes contra los franceses y participó en la batalla de San Quintín (1557) y en 1565, con el título de adelantado y gobernador de la Florida, partió hacia esas tierras con la misión de expulsar a los hugonotes franceses. En 1567, Felipe II le nombró gobernador en Cuba y en 1574 le llamó a España para organizar, como capitán general de la flota, la Armada Invencible, sorprendiéndole la muerte en esta tarea ${ }^{33}$.

La idea de elevar un monumento a su memoria surgió, parece ser, en 1896. Fue promovida por el Marqués de Teverga quien, en una carta dirigida al municipio, reclamaba como "justicia histórica" el atender a la erección de dos monumentos, uno a Pedro Menéndez y otro a Rui Pérez ${ }^{34}$. Fruto de esta denuncia fue el diseño encargado al escultor Cipriano Folgueras en 1900, que no se llegó a realizar ${ }^{35}$.

Esta iniciativa se volvió a emprender quince años después, a través de las páginas del diario local ${ }^{36}$. Oportunamente acogida la proposición, se convocó una reunión para el 9 de septiembre de 1915 en la Ayudantía de Marina ${ }^{37}$. En ella se formaron el Comité Ejecutivo (presidido por el Marqués de Ferrera) y el Comité de Honor encargados de erigir dicho monumento. Previsto en un origen para ser inaugurado en el mes de agosto de 1916, durante las fiestas de San Agustín ${ }^{38}$, contaba con el apoyo del Estado, la Marina de Guerra y la Orden Militar de Santiago, todas relacionadas históricamente con este personaje ${ }^{39}$.

Para sufragar la construcción de la obra se promovió una suscripción popular. Ésta se inició a principios de octubre de 1915 con la cantidad de mil pesetas, aportadas por el Comité Ejecutivo ${ }^{40}$, y se cerró en septiembre de 1916 con un total de veintiocho mil cuatrocientas treinta y nueve pesetas con cincuenta céntimos.

La elevación de este monumento fue acogida a nivel regional e incluso nacional, con lo que se fomentó una mayor difusión del proyecto y recaudación de dinero. A propuesta de David García Somines, la Diputación Provincial contribuyó con dos mil pesetas ${ }^{41}$. Otro

${ }_{33}$ Con motivo de la erección del monumento a su memoria se publicó en La Voz de Avilés una aproximación biográfica, en capítulos publicados entre el día 21-9-1915 y el 14-10-1915. También incluye una breve referencia biográfica José Manuel Paraja en la ficha de este monumento incluida en La estatuaria en Asturias, Oviedo, 1965, p. 17.

${ }^{34}$ Libro de Acuerdos, Año 1896, n. ${ }^{\circ}$ 80, Fols. 85-87, Archivo Municipal de Avilés y El Diario de Avilés, Avilés, 7-11-1896. Cita tomada de MAdrID, J. C. de la, y MADrID, V. de la, Cuando Avilés construyó un teatro, Avilés, 1992, p. 131, edición reeditada y ampliada, Oviedo, 2002, p. 209.

${ }_{35}$ El Diario de Avilés, Avilés, 1-4-1900. Cita tomada de MAdrid, J. C. de la, y Madrid, V. de la, Cuando Avilés..., ob. cit., p. 131.

36 "Por el Monumento a Pedro Menéndez de Avilés", La Voz de Avilés, Avilés, 9-9-1915, p. 2.

37 “Monumento a Pedro Menéndez", El Carbayón, Oviedo, 11-9-1915, p. 2.

38 "Por el Monumento a Pedro Menéndez de Avilés", La Voz de Avilés, Avilés, 11-9-1915, p. 1. La fecha fue posteriormente retrasada a agosto de 1917, La Voz de Avilés, Avilés, 2-7-1916, p. 1, pero en junio de ese año todavía se ponía la primera piedra ( $\mathrm{La}$ Voz de Avilés, Avilés, 16-6-1917) y no se había fundido la estatua (fundición realizada en Codina Hermanos, Madrid, en agosto-septiembre de 1917, Codina Hermanos. Libro de Obras realizadas 1915-1929, libro 2, Fundición Codina, Madrid). Finalmente, fue celebrada en agosto de 1918 con visita de la Infanta Isabel, "Avilés en fiestas. Los solemnes actos de ayer", La Voz de Avilés, Avilés, 24-8-1918, p. 1.

39 “De Avilés. Monumento a Pedro Menéndez", El Carbayón, Oviedo, 25-9-1915, p. 4.

40 "Por el Monumento a Pedro Menéndez de Avilés", La Voz de Avilés, Avilés, 8-10-1915, p. 2.

41 "Por el Monumento a Pedro Menéndez de Avilés", La Voz de Avilés, Avilés, 4-12-1915, p. 2. 
contribuyente destacado, por iniciativa de José Manuel Pedregal, fue el Estado, que sufragó por ley con cinco mil pesetas ${ }^{42}$. También en Oviedo se celebraron varios actos destinados a costear la obra, como un concierto en el teatro Campoamor, un té aristocrático y de pago en el Hotel Covadonga y un festival de carácter popular en la Plaza de Toros ${ }^{43}$. Por otra parte, entre los suscriptores, cuyos nombres y participaciones aparecieron puntualmente publicados en La Voz de Avilés, figuraban, como señala Juan Carlos de la Madrid ${ }^{44}$, numerosos indianos, especialmente cubanos ${ }^{45}$. De hecho, en La Habana se organizó también un comité gestor. Presidido por el avilesino Ramón López Fernández, recaudó en la ciudad y en otras poblaciones cubanas la no despreciable cantidad de trece mil doscientas cincuenta pesetas con cincuenta y cinco céntimos ${ }^{46}$.

Destinado a ocupar la glorieta Norte del Parque del Muelle ${ }^{47}$, no se abrió concurso público, sino que contó con el ofrecimiento desinteresado de varios artistas para modelar los bocetos, de entre los que se eligió el ganador. En un primer momento se ofrecieron Manuel Garci González, Julio González Pola y Víctor Hevia, así como el arquitecto madrileño, de origen asturiano, Benito González del Valle ${ }^{48}$. Al final, se presentaron los proyectos del valenciano Manuel Garci González (fig. 11, quien resultó el ganador ${ }^{49}$, pese a ser su idea la menos innovadora), Víctor Hevia (fig. 12) y el conjunto de Julio Antonio y Sebastián Miranda ${ }^{50}$ (fig. 13).

A diferencia de los ejemplos anteriores, en este caso desconocemos cómo se produjo la asociación entre el escultor tarraconense y el asturiano, ni cuál fue la participación de cada uno. El único documento que se ha localizado que demuestra la autoría conjunta es la reproducción de la maqueta publicada en una revista avilesina ${ }^{51}$, cuyo pie de foto atribuye la autoría a ambos es-

42 Recogida en La Voz de Avilés, Avilés, 1-12-1915 p. 1. Ley aprobada el 16-6-1916, La Voz de Avilés, Avilés, 20-6-1916, p. 1.

43 "Por el Monumento a Pedro Menéndez de Avilés", La Voz de Avilés, Avilés, 5-11-1915, p. 2; 23-11-1915, p. 1; 25-11-1915, p. 1; 28-11-1915, p. 2.

44 Madrid, J. C. de la, y Madrid, V. de la, Cuando Avilés..., ob. cit., p. 134.

45 "Por el Monumento a Pedro Menéndez de Avilés", La Voz de Avilés, Avilés, 30-10-1915, p. 1.

46 Sobre la participación indiana en Cuba se publicaron numerosos artículos: "El monumento a Pedro Menéndez", Asturias. Revista Ilustrada del Centro Asturiano de La Habana, La Habana, 21-1-1916, s/p; 14-3-1916, s/p; 21-5-1916, s/p; 23-7-1916, s/p, y La Voz de Avilés, Avilés, 6-1-1916, p. 1; 23-1-1916, p. 2; 3-2-1916, p. 1; 7-5-1916, p. 2; 5-7-1916, p. 2, 5-9-1916, p. 1.

47 "Por el monumento a Pedro Menéndez de Avilés", La Voz de Avilés, Avilés, 8-12-1916, p. 2. Posteriormente conocido como Parque del General Sanjurjo, Paraja, J. M., Estatuaria..., ob. cit, p. 19, o actualmente Parque del Muelle.

48 "Por el Monumento a Pedro Menéndez de Avilés", La Voz de Avilés, Avilés, 30-10-1915, p. 1.

49 "El monumento a Pedro Menéndez de Avilés. Concurso adjudicado", La Voz de Avilés, Avilés, 30-3-1916, p. 1. A la vez que el boceto ganador, el Comité Ejecutivo también decidía que daría, si el monto de la suscripción lo permitía, premios en metálico a los otros autores, como compensación al trabajo que se han impuesto y como expresión de reconocimiento a su brillante cooperación en el mencionado concurso. No se sabe si este dinero fue efectivamente concedido.

50 "Asturias honrando a sus hijos ilustres. Proyecto de Monumento a Pedro Menéndez de Avilés cuyo autor es el joven escultor ovetense Víctor Hevia", Asturias. Revista ilustrada del Centro Asturiano de La Habana, La Habana, 20-2-1916, s/p, "Del monumento a Pedro Menéndez de Avilés. El boceto de S. Miranda", La Voz de Avilés, Avilés, 20-2-1916, p. 2; "Del monumento a Pedro Menéndez de Avilés. Boceto del Sr. Hevia", La Voz de Avilés, Avilés, 22-21916, p. 1; "Del monumento a Pedro Menéndez de Avilés. Boceto del Sr. García González", La Voz de Avilés, Avilés, 24-2-1916, p. 1. Pese a no mencionarse en este artículo la colaboración con Julio Antonio, en la revista festiva avilesina El Bollu de 1916, ilustrada con fotografías de los proyectos de Miranda y Garci González, sí que consta el nombre de Julio Antonio (dichas dos fotografías han sido publicadas en MADRID, J. C. de la, y MADRID, V. de la, Cuando Avilés..., ob. cit., en sus dos versiones). La fotografía del proyecto de Víctor Hevia se publicó en el citado artículo de la revista Asturias.

${ }^{51}$ El Bollu, Revista avilesina de Festejos, 1916.

AEA, LXXXI, 321, ENERO-MARZO 2008, 49-66, ISSN: 0004-0428 
cultores. No obstante, en la prensa diaria de la localidad el boceto aparece firmado solamente por Sebastián Miranda. El papel concedido a la estructura arquitectónica y el empleo de referencias clásicas refuerzan la hipótesis de la participación de Julio Antonio.

Aunque sólo nos detengamos en el último proyecto, se puede señalar cómo todas las maquetas incluían un basamento y un cuerpo principal, la figura del homenajeado y el escudo de la villa, variedad de materiales según su emplazamiento y un mayor o menor número de referencias alegóricas relacionadas con el efigiado o referencias formales a la antigüedad. Estas características eran explicadas por los propios artistas a través de un texto que acompañaba al modelo. En el caso de la propuesta de Julio Antonio y Sebastián Miranda, y como vemos en la ilustración:

El monumento consta de tres cuerpos: basamento, plinto y obelisco.

El basamento sigue la delineación o perfil de plinto y está construido en granito sin desbastar, al estilo que Vasari llamaba rústico o natural; el plinto es de forma de dado apuntado (sic) la oblicuidad de la pirámide que continúa en el obelisco. Las cuatro aristas laterales están reforzadas por sendas superestructuras cúbicas a manera de contrafuertes, coronadas por guirnaldas de bronce (homenaje de victoria) que penden a entrambos costados del contrafuerte, con carácter funeral. Al frente del plinto se adelanta un zócalo orillado en la línea y ángulos superiores con un elemento decorativo de piedra labrada y dorada, tomada del románico español. En este zócalo va la leyenda epigráfica y sirve de sustento a la estatua, la cual representa al adelantado de Avilés sosteniendo en una mano el áncora símbolo de navegante y en la otra la espada de guerrero y conquistador, con un manto a la usanza de las estatuas clásicas y en actitud de serenidad y fortaleza.

El material de la estatua es bronce, empavonado y dorado. A la espalda del plinto va el escudo de la villa de Avilés labrado en piedra. Sobre el plinto se erige el obelisco, que en su elevación y simplicidad quiere representar el esfuerzo hacia el ideal. Plinto y obelisco son de piedra labrada.

Corona el monumento el águila broncínea con las alas extendidas, noble animal que simboliza la acometividad, la intrepidez, el denuedo, el triunfo y el imperio, virtudes todas que caracterizan la gloriosa epopeya de los conquistadores hispanos.

En las proporciones del monumento se ha buscado compaginar la gracia con la fuerza ${ }^{52}$.

Nos encontramos de nuevo con una serie de características, constantes en las tres obras vistas hasta ahora. En primer lugar, el empleo de contrafuertes en las esquinas, utilizados como refuerzo y motivo ornamental, para dar un mayor juego de volúmenes. Segundo, el contraste de materiales y colores de la piedra, el bronce y los dorados. Y por último, la referencia a motivos clásicos, tanto formales como iconográficos. El obelisco, elegido como eje principal del monumento, es un elemento de clara tradición egipcia y clásica, que contaba además con numerosos antecedentes en esculturas conmemorativas en España, como son el madrileño monumento a Los héroes del 2 de mayo, de 1822, diseñado por Isidro González Velázquez, el barcelonés Monumento a Francesc de Paula Rius y Taulet, diseñado por Manuel Fuxá y Eusebio Arnau en 1896, y el proyecto inicial para el Monumento a Fernando Villaamil, en Castropol (Asturias) de Cipriano Folgueras, de 1907. Los tres pudieron influir en este boceto, en especial el madrileño, en donde el obelisco también se relaciona con figuras heroicas, aunque en el caso de la maqueta avilesina el resto de la composición sea mucho más sencilla y se pierda el carácter retórico de los otros tres modelos.

52 "Del monumento a Pedro Menéndez de Avilés. El boceto de S. Miranda", La Voz de Avilés, Avilés, 20-2-1916, p. 2.

AEA, LXXXI, 321, ENERO-MARZO 2008, 49-66, ISSN: 0004-0428 


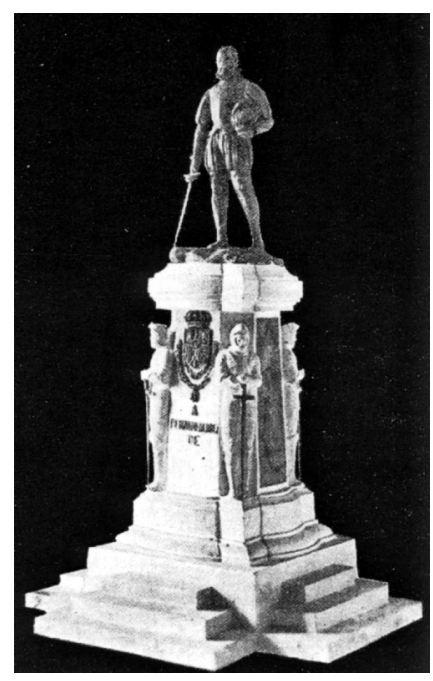

Fig. 11. Manuel Garci González. Proyecto del Monumento a Pedro Menéndez de Avilés (1916).

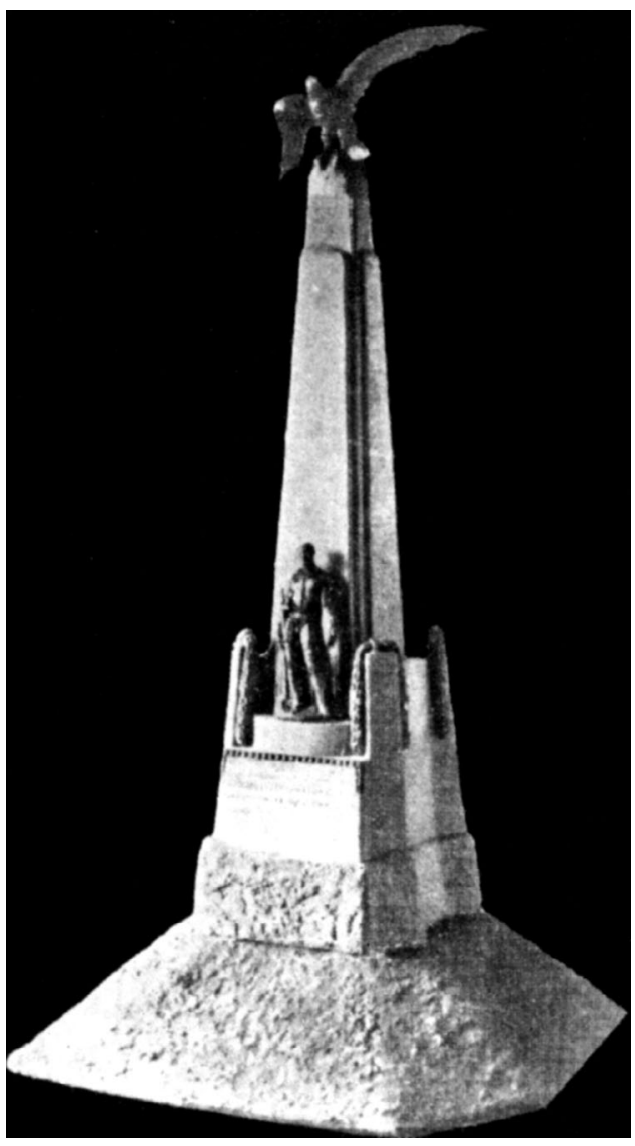

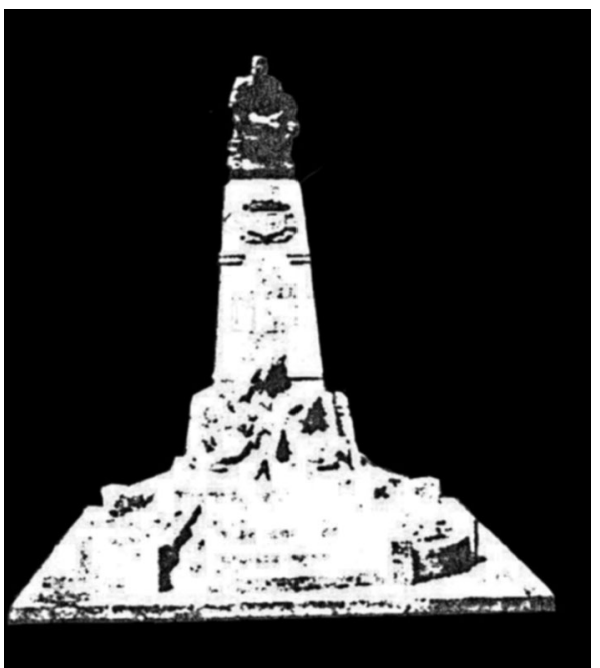

Fig. 12. Víctor Hevia.

Proyecto para el Monumento a Pedro Menéndez de Avilés (1916).
Fig. 13. Julio Antonio y Sebastián Miranda. Proyecto para el Monumento a Pedro Menéndez de Avilés (1916). 
Motivos iconográficos de tradición clásica son las guirnaldas, el ánfora, el manto y el águila de coronación. Sorprende aquí el empleo de un elemento tomado del románico español, como es el del motivo decorativo de piedra labrada y dorada, que recuerda a un ajedrezado jaqués. El ambiente de bohemia y su interés por los viajes dentro y fuera de la Península, característico de ambos escultores y que se reflejó en sus retratos de distintos personajes y razas de España, quedó aquí plasmado en una manifestación de conocimiento del arte del propio país. Este motivo románico, unido a elementos de la Grecia clásica, se equipara en dignidad y tradición con ellos.

En cuanto a la efigie de Pedro Menéndez de Avilés, caracterizado como navegante, héroe y conquistador, pudiera estar tomada del dibujo de Josef Camarón que, grabado por Francisco de Paula Martí, formó parte de la colección de Retratos de españoles ilustres con un epítome de sus vidas, publicada en Madrid en 1791.

Los autores continuaron buscando, en este proyecto como en los anteriores, una renovación de la idea del monumento conmemorativo a través de la depuración de las formas, de la recuperación de la tradición española y clásica y de un simbolismo alegórico de raíz también clásica.

\section{Epílogo de una colaboración}

De lo expuesto hasta ahora se pueden extraer varias conclusiones sobre el trabajo común de Julio Antonio y Sebastián Miranda. Parece que su colaboración fue circunstancial: salvo para el tiempo que trabajaron en el proyecto del Monumento a las Américas (aproximadamente desde el último trimestre de 1913 al primero de 1914), en que compartieron taller, sus otras dos producciones conjuntas fueron uniones puntuales. De hecho, y pese a la amistad mutua y profunda admiración del asturiano por el catalán, cada uno de estos dos escultores mantuvo sus viajes, proyectos, asociaciones con otros artistas y carreras independientes, y ninguno de los dos se vio formal o estilísticamente influido por el otro. Así, Miranda continuó con su escultura caricaturesca de pequeño formato y Julio Antonio con sus retratos realistas y sus proyectos monumentales.

Las maquetas realizadas, y sobre todo la de América, fueron ideas de gran monumentalidad, en la línea de los trabajos de Julio Antonio. En ellas, las arquitecturas adquirían gran importancia y desarrollo, integrándose con la escultura, y el realismo de las figuras dejaba paso al triunfo de las alegorías y los símbolos. A la par, se buscaba una recuperación de la antigüedad clásica y nacional, elementos con los que se alejaba de la concepción decimonónica del monumento público que todavía triunfaba en España, hecho que repercutió en el poco éxito de los mismos.

Por último, y pese a la puesta de ideas en común, de la concepción general de los monumentos se puede deducir que el peso creador lo llevaba Julio Antonio. Su formación, referencias e ideas eran en muchos puntos coincidentes, pero la grandiosidad y ambición de los diseños está mucho más próxima al resto de la producción julioantoniana que a la de Miranda. Lamentablemente estos proyectos, como la mayoría de las utopías monumentales diseñadas por Julio Antonio en solitario, siguieron su misma desafortunada suerte, no llegando a construirse o sufriendo graves modificaciones. Ello no es óbice para considerar su interés, su calidad, y su papel en la renovación formal del monumento conmemorativo en el primer tercio del siglo XX en España.

Fecha de recepción: 2-VI-2006

Fecha de aceptación: 15-X-2006

AEA, LXXXI, 321, ENERO-MARZO 2008, 49-66, ISSN: 0004-0428 